\title{
Characterizing the Effects of Site-Specific Metastases on Nasopharyngeal Squamous Cell Carcinoma Survival
}

\section{Christopher C Tseng ${ }^{1}$, Jeff Gao ${ }^{1}$, Gregory L Barinsky ${ }^{1}$, Jordon G Grube $^{2}$, Christina H Fang ${ }^{3}$, Jean Anderson Eloy ${ }^{1,4,5,6,7}$ and Wayne D Hsueh $^{1,4 *}$}

${ }^{1}$ Department of Otolaryngology - Head and Neck Surgery, Rutgers New Jersey Medical School, Newark, New Jersey, USA

${ }^{2}$ Department of Otolaryngology, Albany Medical Center, Albany, New York, USA ${ }^{3}$ Department of Otorhinolaryngology - Head and Neck Surgery, Montefiore Medical Center, The University Hospital of Albert Einstein College of Medicine, Bronx, New York, USA

${ }^{4}$ Center for Skull Base and Pituitary Surgery, Neurological Institute of New Jersey, Rutgers New Jersey Medical School, Newark, New Jersey, USA

${ }^{5}$ Department of Neurological Surgery, Rutgers New Jersey Medical School, Newark, New Jersey, USA

${ }^{6}$ Department of Ophthalmology and Visual Science, Rutgers New Jersey Medical School, Newark, New Jersey, USA

${ }^{7}$ Department of Otolaryngology and Facial Plastic Surgery, Saint Barnabas Medical Center - RWJ Barnabas Health, Livingston, New Jersey, USA

*Corresponding Author: Wayne D Hsueh, Assistant Professor, Department of Otolaryngology - Head and Neck Surgery, Rutgers New Jersey Medical School, Newark, New Jersey, USA.

\section{Received: August 25, 2021}

Published: September 11, 2021

(C) All rights are reserved by Wayne D Hsueh., et al.

\footnotetext{
Abstract

Introduction: To investigate the impact of site-specific metastases on survival and identify predictors of metastasis in patients with nasopharyngeal squamous cell carcinoma (SCC).

Materials and Methods: The National Cancer Database was queried for all cases of nasopharyngeal SCC between 2010-2016 which had metastasized to distant sites (460 cases). This was then further classified as metastases to bone, liver, lung, brain, and multiple sites. Univariate and multivariate analyses were used to compare patient demographics between sites. Kaplan-Meier analysis was applied to compare overall survival.
}

Results: A total of 145 (31.5\%) distant metastases to bone, 55 (12.0\%) to liver, 112 (24.3\%) to lung, 15 (3.5\%) to brain, and 113 $(28.9 \%)$ to multiple sites were identified. One-year survival for the entire cohort was $50.4 \%$ with a median survival of 12.4 months. One-year survival was $58.3 \%$ for liver metastasis, $53.2 \%$ for bone, $48.9 \%$ for multiple sites, $44.2 \%$ for brain, and $42.7 \%$ for lung (logrank $p=0.252)$. Patient age $(p=0.009)$ and treatment facility type $(p=0.019)$ were found to predict the site of distant metastasis. Being younger than 60 had lower odds of metastasis to liver compared to bone (OR 0.527, 95\% CI [0.311 - 0.893], p = 0.017).

Conclusion: There was no significant difference found in survival between metastases to different sites. The patient's treatment facility type and age were associated significantly with lower odds of nasopharyngeal SCC metastasis to certain distant sites.

Keywords: Nasopharynx; National Cancer Database; Squamous Cell Carcinoma, Survival, Metastasis, Distant Site 


\section{Abbreviations}

NCDB: National Cancer Database; SCC: Squamous Cell Carcinoma; ICD-0-3: International Classification of Diseases for Oncology, $3^{\text {rd }}$ Edition

\section{Introduction}

Nasopharyngeal carcinoma is an uncommon head and neck malignancy, although it has a notably distinct geographic distribution. Overall, nasopharyngeal carcinoma composes less than 1 per 100,000 cancer cases worldwide, but upwards of 30 per 100,000 cancer cases in endemic regions such as southern China [1,2]. Unique nasopharyngeal carcinoma histologies also display significant regional dependence. Keratinizing squamous cell carcinoma (SCC), while identified in $2 \%$ of nasopharyngeal carcinoma cases in southern China, can be found in $25 \%$ of cases in North America [2]. As a result, specifically analyzing characteristics of a major histologic subtype of nasopharyngeal carcinoma may more precisely define prognostic factors and treatment approaches. This has relevancy in regions of the world in which patients present more frequently with these specific histologies.

Nasopharyngeal SCC is associated with significant morbidity and mortality, including cranial nerve involvement, intracranial extension, and a poor overall 5-year survival rate of 32.3\% [3,4]. Several factors have been found to play a role in prognosis, including tumor stage and size, locoregional invasion such as cranial nerve involvement, and distant spread [2,4-6]. Due to its anatomic location, most nasopharyngeal SCCs are diagnosed late in the disease course, with $88.5 \%$ of patients diagnosed with stage III or IV cancer on initial presentation [4]. Furthermore, nasopharyngeal SCC has been noted to rapidly metastasize to regional cervical nodes. Distant metastases has been shown to increase the risk of disease relapse and worsen survival [7-9]. SCC of the nasopharynx has increased propensity to spread to distant regions compared to SCC of other primary sites. Altun., et al. noted nodal involvement in $75 \%$ - $90 \%$ of cases, along with $38 \%-87 \%$ of nasopharyngeal SCC cases showing evidence of distant metastases [10]. While the presence of distant metastases is intrinsically a negative prognostic indicator [11], specific metastatic sites have been shown to carry their own individual impact on overall survival and disease management choices, including palliative interventions $[12,13]$. Of note, a study by Qu., et al. examined 389 cases of metastatic nasopharyngeal SCC from the Surveillance, Epidemiology, and End Results database to determine patterns of metastasis to distant sites and significant prognostic indicators for nasopharyngeal SCC patients with distant metastases [14]. While individual distant sites were analyzed and demonstrated that bone, liver, and lung metastasis were especially associated with poor survival, the prognostic implications of each individual site were assessed relative to no metastasis or to nodal metastasis, instead of direct comparison with other sites of distant metastasis. Moreover, patient demographic and clinicopathologic characteristics significantly associated with each distant metastatic site have not been explored, factors which can potentially play a role in more individualized selection of treatment modalities and are thus important to identify. As a result, this study seeks to further investigate the impact of site-specific metastases on survival and identify predictors of metastasis to individual distant sites in patients with nasopharyngeal SCC.

\section{Materials and Methods}

Data source

All cases were gathered from the National Cancer Database (NCDB), a Commission on Cancer joint effort from the American College of Surgeons and the American Cancer Society to record cancer cases from over 1,500 cancer facilities in the United States, with data on approximately 34 million cancer cases nationwide [15]. The American College of Surgeons and the Commission on Cancer have not verified and are therefore not responsible for the conclusions drawn from analysis of this data by the investigator. De-identified data from the NCDB was used for this study and are considered exempt from Rutgers New Jersey Medical School institutional review board review.

\section{Study population}

The NCDB was queried for cases of nasopharyngeal SCC in 2010-2016. Patients with primary site codes for the nasopharynx were selected using the International Classification of Diseases for Oncology, $3^{\text {rd }}$ Edition (ICD-0-3) topography codes C11.0, C11.1, C11.2, C11.3, C11.8, C11.9. Patients with a histology code for squamous cell carcinoma were selected using ICD-0-3 histology codes 8070, 8071, 8072, 8073, 8074, 8076, 8078. Non-malignant cases which did not metastasize to distant sites were excluded. Site-specific metastases were stratified into distant metastasis to the bone only, to the liver only, to the lung only, to the brain only, or to multiple distant sites. Patient demographic and clinical variables examined included patient age, sex, race, Charlson-Deyo score, treatment facility type, insurance status, histology, and clinical $\mathrm{N}$ stage. While additional tumor characteristics including grade, clinical $\mathrm{T}$ stage, and tumor size were examined, they were excluded from final analyses due to no recorded values for metastasis to certain distant sites. 


\section{Statistical analysis}

For univariate analysis and cross-tabulation, Pearson's chisquare and Fisher's exact test were utilized as appropriate. Multivariate logistic regression was then performed to determine association between factors with $\mathrm{p}<0.05$ on univariate analysis and site-specific metastases, using metastasis to bone as a reference. Overall survival for site-specific metastases was calculated using the Kaplan-Meier method. Statistical significance for all tests was set at an alpha level of $<0.05$, and all statistical analysis was performed using SPSS 24 (IBM, Armonk, New York).

\section{Results}

A total of 460 cases of nasopharyngeal SCC which metastasized to distant sites were identified in our study population, with 145 (31.5\%) cases of metastases to bone, 55 (12.0\%) to liver, 112 (24.3\%) to lung, 15 (3.5\%) to brain, and 133 (28.9\%) to multiple sites. Table 1 shows univariate analysis of patient demographic and clinical characteristics. The majority of patients in our study cohort were under 60 years old (52.0\%), male (76.7\%), and white $(62.0 \%)$. Age $(p=0.009)$ and treatment facility type $(p=0.019)$ were significantly associated with nasopharyngeal SCC distant metastasis at the time of diagnosis. While nasopharyngeal SCC patients with metastasis to bone, liver, brain, or multiple sites were more likely to be under 60 years old, those with metastasis to lung were more likely to be at least 60 years of age or older. Moreover, a greater proportion of nasopharyngeal SCC patients with distant metastasis to bone, liver, or brain were treated at a community cancer program, whereas a greater proportion of patients with distant metastasis to lung or multiple sites were treated at an academic/ research program. Patient's sex, race, Charlson-Deyo score, insurance status, histology, and clinical $\mathrm{N}$ stage were found to not be significantly associated with distant metastases. A multivariate logistic regression model for each metastatic site (or sites) was built with variables found to be significant on univariate analysis (Table 2). Nasopharyngeal SCC patients with metastasis to lung were less likely to be treated at a community cancer program (OR 0.421, 95\% CI [0.178 - 0.995], $\mathrm{p}=0.049$ ), while metastasis to brain were less likely to be treated at an academic/research program (OR 0.045, $95 \% \mathrm{CI}$ [0.005 - 0.439], $\mathrm{p}=0.008$ ). Metastasis to the liver were less likely to be under 60 years old (OR 0.527, 95\% CI [0.311 - 0.893], $\mathrm{p}=0.017$ ), compared to patients with metastasis to bone. There were no statistically significant associations between patient age and facility type with distant metastases to multiple sites on multivariate analysis. Following Kaplan-Meier survival analysis, one-year overall survival for all patients in our study cohort was $50.42 \%$ with a median survival of 12.42 months (Table 3 ). Oneyear survival for patients with metastasis to bone was $53.20 \%$ (logrank $\mathrm{p}=0.141$ ); to liver was $58.32 \%$ ( $\log$-rank $\mathrm{p}=0.363$ ); to lung was $42.65 \%$ (log-rank $p=0.252$ ); to brain was $44.22 \%$ (log-rank $\mathrm{p}=0.674$ ); and to multiple distant sites was $48.86 \%$ (log-rank $\mathrm{p}=$ 0.172 ). Site-specific metastases were found to not be significantly associated with survival.

\begin{tabular}{|c|c|c|c|c|c|c|c|}
\hline Variable & $\begin{array}{c}\text { Bone } \\
(N=145)\end{array}$ & $\begin{array}{c}\text { Liver } \\
(N=55)\end{array}$ & $\begin{array}{c}\text { Lung } \\
(\mathrm{N}=112)\end{array}$ & $\begin{array}{c}\text { Brain } \\
(N=15)\end{array}$ & $\begin{array}{l}\text { Multiple Sites } \\
\qquad(\mathrm{N}=113)\end{array}$ & Total $(N=460)$ & p-value \\
\hline Age, years & & & & & & & 0.009 \\
\hline$<60$ & $78(53.8 \%)$ & $34(61.8 \%)$ & $42(37.5 \%)$ & $9(60.0 \%)$ & $76(57.1 \%)$ & $239(52.0 \%)$ & \\
\hline $60+$ & $67(46.2 \%)$ & $21(38.2 \%)$ & $70(62.5 \%)$ & $6(40.0 \%)$ & $57(42.9 \%)$ & $221(48.0 \%)$ & \\
\hline Sex & & & & & & & 0.307 \\
\hline Male & $104(71.7 \%)$ & $45(81.8 \%)$ & $92(82.1 \%)$ & $11(73.3 \%)$ & $101(75.9 \%)$ & $353(76.7 \%)$ & \\
\hline Female & $41(28.3 \%)$ & $10(18.2 \%)$ & $20(17.9 \%)$ & $4(26.7 \%)$ & $32(24.1 \%)$ & $107(23.3 \%)$ & \\
\hline Race & & & & & & & 0.643 \\
\hline White & $93(64.1 \%)$ & $32(58.2 \%)$ & $74(66.1 \%)$ & $11(73.3 \%)$ & $75(56.4 \%)$ & $285(62.0 \%)$ & \\
\hline Black & $24(16.6 \%)$ & $9(16.4 \%)$ & $21(18.8 \%)$ & $4(26.7 \%)$ & $25(18.8 \%)$ & $83(18.0 \%)$ & \\
\hline Asian/Pacific Islander & $23(15.9 \%)$ & $13(23.6 \%)$ & $13(11.6 \%)$ & $0(0.0 \%)$ & $29(21.8 \%)$ & $78(17.0 \%)$ & \\
\hline Other & $4(2.8 \%)$ & $0(0.0 \%)$ & $2(1.8 \%)$ & $0(0.0 \%)$ & $2(1.5 \%)$ & $8(1.7 \%)$ & \\
\hline Unknown & $1(0.7 \%)$ & $1(1.8 \%)$ & $2(1.8 \%)$ & $0(0.0 \%)$ & $2(1.5 \%)$ & $6(1.3 \%)$ & \\
\hline Charlson-Deyo Score & & & & & & & 0.344 \\
\hline 0 & $120(82.8 \%)$ & $45(81.8 \%)$ & $83(74.1 \%)$ & $10(66.7 \%)$ & $110(82.7 \%)$ & $368(80.0 \%)$ & \\
\hline 1 & $15(10.3 \%)$ & $6(10.9 \%)$ & $21(18.8 \%)$ & $2(13.3 \%)$ & $15(11.3 \%)$ & $59(12.8 \%)$ & \\
\hline
\end{tabular}




\begin{tabular}{|c|c|c|c|c|c|c|c|}
\hline $2+$ & $10(6.9 \%)$ & $4(7.3 \%)$ & $8(7.1 \%)$ & $3(20.0 \%)$ & $8(6.0 \%)$ & $33(7.2 \%)$ & \\
\hline Facility Type & & & & & & & 0.019 \\
\hline Community Program & $62(46.3 \%)$ & $26(55.3 \%)$ & $42(38.9 \%)$ & 7 (58.3\%) & $48(39.7 \%)$ & $185(43.8 \%)$ & \\
\hline $\begin{array}{l}\text { Academic/Research Pro- } \\
\text { gram }\end{array}$ & $61(45.5 \%)$ & $18(38.3 \%)$ & 49 (45.4\%) & $1(8.3 \%)$ & $62(51.2 \%)$ & $191(45.3 \%)$ & \\
\hline $\begin{array}{l}\text { Integrated Network Cancer } \\
\text { Program }\end{array}$ & $11(8.2 \%)$ & $3(6.4 \%)$ & $17(15.7 \%)$ & $4(33.3 \%)$ & $11(9.1 \%)$ & 46 (10.9\%) & \\
\hline Insurance Status & & & & & & & 0.869 \\
\hline Private Insurance & $56(38.6 \%)$ & $23(41.8 \%)$ & $36(32.1 \%)$ & $6(40.0 \%)$ & $43(32.3 \%)$ & $164(35.7 \%)$ & \\
\hline Uninsured & $16(11.0 \%)$ & $7(12.7 \%)$ & $12(10.7 \%)$ & $0(0.0 \%)$ & $18(13.5 \%)$ & $53(11.5 \%)$ & \\
\hline Government & $70(48.3 \%)$ & $24(43.6 \%)$ & $60(53.6 \%)$ & $8(53.3 \%)$ & 69 (51.9\%) & $231(50.2 \%)$ & \\
\hline Unknown & $3(2.1 \%)$ & $1(1.8 \%)$ & $4(3.6 \%)$ & $1(6.7 \%)$ & $3(2.3 \%)$ & $12(2.6 \%)$ & \\
\hline Histology & & & & & & & 0.077 \\
\hline $\begin{array}{l}\text { Keratinizing squamous cell } \\
\text { carcinoma }\end{array}$ & $95(65.5 \%)$ & $33(60.0 \%)$ & $86(76.8 \%)$ & $13(86.7 \%)$ & $94(70.7 \%)$ & $321(69.8 \%)$ & \\
\hline $\begin{array}{l}\text { Non-keratinizing squamous } \\
\text { cell carcinoma }\end{array}$ & $50(34.5 \%)$ & $22(40.0 \%)$ & $26(23.2 \%)$ & $2(13.3 \%)$ & $39(29.3 \%)$ & $139(30.2 \%)$ & \\
\hline Clinical N Stage & & & & & & & 0.075 \\
\hline 0 & $22(19.8 \%)$ & $1(2.6 \%)$ & $12(14.0 \%)$ & $5(38.5 \%)$ & $10(12.3 \%)$ & $50(15.2 \%)$ & \\
\hline 1 & $23(20.7 \%)$ & $14(35.9 \%)$ & $29(33.7 \%)$ & $1(7.7 \%)$ & $24(29.6 \%)$ & $91(27.6 \%)$ & \\
\hline 2 & $56(50.5 \%)$ & $20(51.3 \%)$ & $42(48.8 \%)$ & $6(46.2 \%)$ & $41(50.6 \%)$ & $165(50.0 \%)$ & \\
\hline 3 & $10(9.0 \%)$ & $4(10.3 \%)$ & $3(3.5 \%)$ & $1(7.7 \%)$ & $6(7.4 \%)$ & $24(7.3 \%)$ & \\
\hline
\end{tabular}

Table 1: Patient demographic, tumor, and hospital characteristics stratified by site of distant metastasis. Bolded values signify statistical significance $(\mathrm{p}<0.05)$.

\begin{tabular}{|l|c|c|c|}
\hline Metastasis to Lung & \multicolumn{2}{|l|}{} & \\
\hline Facility Type & Odds Ratio & $\mathbf{9 5 \%}$ Confidence Interval & p-value \\
\hline Community Cancer Program & 0.421 & $0.178-0.995$ & $\mathbf{0 . 0 4 9}$ \\
\hline Academic/Research Program & 0.542 & $0.231-1.272$ & 0.159 \\
\hline Integrated Network Cancer Program & Reference & & \\
\hline Age, years & & & 0.447 \\
\hline$<60$ & 1.299 & $0.662-2.548$ & \\
\hline $60+$ & Reference & & p-value \\
\hline Metastasis to Liver & & & 0.518 \\
\hline Facility Type & Odds Ratio & $\mathbf{9 5 \%}$ Confidence Interval & 0.930 \\
\hline Community Cancer Program & 1.565 & $0.403-6.085$ & \\
\hline Academic/Research Program & 1.064 & $0.267-4.237$ & $\mathbf{0 . 0 1 7}$ \\
\hline Integrated Network Cancer Program & Reference & & \\
\hline Age, years & & & \\
\hline$<60$ & 0.527 & $0.311-0.893$ & \\
\hline $60+$ & Reference & & \\
\hline
\end{tabular}




\begin{tabular}{|c|c|c|c|}
\hline \multicolumn{4}{|l|}{ Metastasis to Brain } \\
\hline Facility Type & Odds Ratio & 95\% Confidence Interval & p-value \\
\hline Community Cancer Program & 0.313 & $0.078-1.256$ & 0.101 \\
\hline Academic/Research Program & 0.045 & $0.005-0.439$ & 0.008 \\
\hline Integrated Network Cancer Program & Reference & & \\
\hline \multicolumn{4}{|l|}{ Age, years } \\
\hline$<60$ & 1.141 & $0.343-3.791$ & 0.830 \\
\hline $60+$ & Reference & & \\
\hline \multicolumn{4}{|l|}{ Metastasis to Multiple Sites } \\
\hline Facility Type & Odds Ratio & 95\% Confidence Interval & p-value \\
\hline Community Cancer Program & 0.779 & $0.311-1.949$ & 0.593 \\
\hline Academic/Research Program & 1.011 & $0.408-2.506$ & 0.981 \\
\hline Integrated Network Cancer Program & Reference & & \\
\hline \multicolumn{4}{|l|}{ Age, years } \\
\hline$<60$ & 1.088 & $0.662-1.788$ & 0.740 \\
\hline $60+$ & Reference & & \\
\hline
\end{tabular}

Table 2: Logistic regression of factors associated with metastasis to distant sites (Reference to metastasis to bone).

Bolded values signify statistical significance $(\mathrm{p}<0.05)$.

\begin{tabular}{|l|c|c|c|}
\hline Site & $\begin{array}{c}\text { 1-Year Overall } \\
\text { Survival }\end{array}$ & $\begin{array}{c}\text { Median Survival } \\
\text { (months) }\end{array}$ & Log-rank \\
\hline Bone & $53.20 \%$ & 13.21 & 0.141 \\
\hline Liver & $58.32 \%$ & 16.95 & 0.363 \\
\hline Lung & $42.65 \%$ & 9.53 & 0.252 \\
\hline Brain & $44.22 \%$ & 13.17 & 0.674 \\
\hline Multiple & $48.86 \%$ & 11.93 & 0.172 \\
\hline Cohort & $50.42 \%$ & 12.42 & \\
\hline
\end{tabular}

Table 3: Kaplan Meier survival stratified by site of distant metastasis.

\section{Discussion}

The finding of distant metastasis is known to carry a negative prognosis for malignancies, where specific distant sites may be indications for different treatment modalities and convey varying effects on overall survival [16]. While nasopharyngeal SCC is a relatively uncommon cancer, it is a significant contributor to morbidity and mortality, with distant metastases recognized as an important reason for treatment failure [17]. Along these lines, this study seeks to better understand the prognostic implications of site-specific metastases in nasopharyngeal SCC patients and elucidate factors associated with metastasis to those distant sites.
The majority of patients with nasopharyngeal SCC in our study population were white males less than 60 years old, consistent with known nasopharyngeal SCC epidemiology in North America $[1,4,8]$. Upon univariate analysis, only patient age and treatment facility type were significantly associated with distant metastases. Subsequent multivariate logistic regression showed that nasopharyngeal SCC patients with lung metastasis were less likely to be treated at a community cancer program than nasopharyngeal SCC patients with bone metastasis. Considering that bone metastasis is the most common distant metastasis for nasopharyngeal carcinomas and is not significantly associated with a worse prognosis compared to other distant sites, it seems evident that nasopharyngeal SCC cases with less frequently encountered lung metastasis were more likely to be treated at an academic/research facility [18-20]. Academic/research facilities are usually more likely to employ technically complex multimodal treatments and offer more comprehensive disease management and individualized care, all of which can lead to improved outcomes for cancer patients [2124]. In addition, Hui., et al. found that nasopharyngeal SCC patients with isolated metastatic disease to the lung can actually have a better prognosis compared to other metastatic sites if an aggressive, multimodal treatment approach is applied, even though the presence of lung metastasis typically portends a worse clinical outlook [17]. As a result, these patients may be more likely to be treated at 
an academic/research facility capable of administering such treatments that improve overall survival, making nasopharyngeal SCC patients with lung metastasis less likely to be treated at a community program. Furthermore, being younger than 60 years old has lower likelihood of liver metastasis compared to bone metastasis, likely because a less common metastatic site such as the liver would be a late finding of advanced head and neck cancers and therefore more likely to present in an older patient. Finally, being treated at an academic/research facility was associated with lower odds of brain metastasis compared to bone metastasis. Identification of brain metastasis typically signifies an especially poor prognosis when diagnosed for a wide variety of malignancies, though it does not have significant prognostic value compared to our distant sites of nasopharyngeal SCC metastasis based on our findings $[25,26]$. Moreover, brain metastasis from nasopharyngeal SCC was the least common of the specific primary sites studied in our cohort, with only 15 total cases recorded, compared to 145 cases of bone metastasis. Due to its low incidence, brain metastasis may be infrequently recognized in nasopharyngeal SCC patients, who also may further present as poor surgical candidates due to low survival rates even following treatment [27]. Thus, these patients may have lower odds of being treated at an academic/research facility compared to patients with bone metastasis.

The most frequent site of distant metastasis for nasopharyngeal SCC in our study was to the bone, followed by metastasis to the lung and to the liver, which is consistent with known patterns of distant metastases among nasopharyngeal carcinomas [19,20]. Our results showed no significant difference in survival between nasopharyngeal SCC metastases to the various distant sites investigated in our study. These findings seem to run contrary to the current literature on nasopharyngeal carcinoma which has indicated that certain distant sites are associated with statistically significant differences in survival, such as lung only metastasis, liver only metastasis, and metastases to multiple sites [28,29]. It should be noted that the prognostic impact of an individual metastatic site can vary in the current literature. In their study examining predominantly WHO type II/III nasopharyngeal SCC patients, Hui., et al. determined that lung only metastasis had an improved prognosis compared to other distant sites [17]. However, when examining a similar patient population, Ong., et al. instead found that lung metastasis was independently associated with a negative prognosis [19]. As our study showed that no individual site of distant metastasis led to significantly worse outcomes compared to other sites, these metastatic sites may not actually have prognostic significance for nasopharyngeal SCC, findings which require further investigation.
Moreover, our results indicate that lung only metastasis had the lowest overall survival compared to the other metastatic sites, though not statistically significant by log-rank. This may suggest that while there are known treatment approaches such as aggressive chemotherapy which can improve outcomes for certain nasopharyngeal carcinoma patients presenting with lung metastasis [17], nasopharyngeal SCC patients may not necessarily always receive the optimal treatment modalities or treating facilities may not have the capability to administer them, hence improved survival for these patients may have yet to be fully realized. As inadequate treatment for metastatic disease can lead to worse overall survival rates, it is critical that appropriate treatment modalities be selected for these patients following diagnosis. In addition, while not statistically significant by log-rank, liver only metastasis was found to have the highest overall survival, though it conveys a negative prognosis in most other studies $[19,28]$. Consequently, specific metastatic sites may potentially have a differential impact on survival for nasopharyngeal SCCs in particular than for nasopharyngeal carcinomas in general, though considering no metastatic site established a statistically significant difference in survivorship, it remains difficult to draw such conclusions at present. Overall, despite recent advances in developing improved treatment approaches, head and neck SCCs continue to maintain a poor prognosis, especially at the advanced stage of distant metastases [30]. While most research on cancers of the nasopharynx has been focused on nasopharyngeal carcinomas in general, studying nasopharyngeal SCC specifically has clinical significance as individual histologies in head and neck malignancies can be associated with varying effects on survival $[31,32]$. Our findings further illustrate the need for future studies to explore risk factors associated with distant metastases and their accompanying prognostic implications for nasopharyngeal SCC patients.

The limitations inherent to studying large databases like the NCDB should be noted. There remains the possibility of inaccuracies recorded in the database due to miscoding made during data entry, as we cannot verify the validity of those entries ourselves. In addition, though a diverse range of variables are recorded in the NCDB, potentially significant factors such as cancer recurrence are missing. Since the cases for this study were from a database instead of from chart review, such details could not be retrieved, and our exploration of long-term patient outcomes was limited to overall survival. Relapse following treatment has a known significant negative impact on survival for nasopharyngeal carcinoma patients, so not being able to include recurrence status may be an important attribute that our analysis on prognostic factors was not 
able to take into consideration. While one should be mindful of associated limitations when interpreting these findings, the capability to investigate the advanced stage of a relatively rare cancer and accompanying demographic and clinicopathologic characteristics on a population-level scale demonstrates the clinical utility of the NCDB for assessing the prognostic value of distant metastatic sites for nasopharyngeal SCC.

\section{Conclusion}

In this study, we determined that nasopharyngeal SCC patient's age and their treatment facility type were factors associated with metastases to certain distant sites. Specifically, being treated at a community cancer program reduced the odds of lung metastasis, being treated at an academic/research program reduced the odds of brain metastasis and being younger than 60 years old reduced the odds of liver metastasis, all relative to bone metastasis. Upon further analysis, we found no significant survival difference between metastases to distant sites. As a result, further research is needed to better clarify the prognostic significance of distant metastases and other factors associated with metastatic nasopharyngeal SCC.

\section{Conflict of Interest}

The authors declare no conflicts of interest or financial disclosures.

\section{Bibliography}

1. Chang ET and Adami HO. "The enigmatic epidemiology of nasopharyngeal carcinoma”. Cancer Epidemiology Biomarkers and Prevention 15.10 (2006): 1765-1777.

2. Wei WI and Sham JS. "Nasopharyngeal carcinoma". The Lancet 365.9476 (2005): 2041-2054.

3. Moloy PJ., et al. "Squamous carcinoma of the nasopharynx". Western Journal of Medicine 143.1 (1985): 66.

4. Farias TP., et al. "Prognostic Factors and Outcome for Nasopharyngeal Carcinoma". Archives of Otolaryngology-Head and Neck Surgery 129.7 (2003): 794-799.

5. Vokes EE., et al. "Nasopharyngeal carcinoma". The Lancet 350.9084 (1997): 1087-1091.

6. Brennan B. "Nasopharyngeal carcinoma". Orphanet Journal of Rare Diseases 1.1 (2006): 1-5.
7. Razak ARA., et al. "Nasopharyngeal carcinoma: The next challenges”. European Journal of Cancer 46.11 (2010): 1967-1978.

8. Patel TD., et al. "Utility of Surgery/Radiotherapy in Distant Metastatic Head and Neck Squamous Cell Carcinoma:A Population-Based Approach". Otolaryngology-Head and Neck Surgery 154.5 (2016): 868-874.

9. Alvi A and Johnson JT. "Development of distant metastasis after treatment of advanced-stage head and neck cancer". Head and Neck: Journal for the Sciences and Specialties of the Head and Neck 19.6 (1997): 500-505.

10. Altun M., et al. "Undifferentiated nasopharyngeal cancer (UCNT): current diagnostic and therapeutic aspects". International Journal of Radiation Oncology* Biology* Physics 32.3 (1995): 859-877.

11. Tian Y-M., et al. "Prognostic model and optimal treatment for patients with stage IVc nasopharyngeal carcinoma at diagnosis". Scientific Reports 9.1 (2019): 19272.

12. Tan HL., et al. "Metastatic gastric cancer: Does the site of metastasis make a difference?" Asia-Pacific Journal of Clinical Oncology 15.1 (2019): 10-17.

13. McClure SA., et al. "Maxillofacial metastases: a retrospective review of one institution's 15-year experience". Journal of Oral and Maxillofacial Surgery 71.1 (2013): 178-188.

14. Qu W., et al. "Pattern and prognosis of distant metastases in nasopharyngeal carcinoma: A large-population retrospective analysis". Cancer Medicine 9.17 (2020): 6147-6158.

15. Bilimoria KY., et al. "The National Cancer Data Base: a powerful initiative to improve cancer care in the United States". Annals of Surgical Oncology 15.3 (2008): 683-690.

16. Chaffer CL and Weinberg RA. "A Perspective on Cancer Cell Metastasis". Science 331.6024 (2011): 1559-1564.

17. Hui EP., et al. "Lung metastasis alone in nasopharyngeal carcinoma: a relatively favorable prognostic group: a study by the Hong Kong Nasopharyngeal Carcinoma Study Group". Cancer 101.2 (2004): 300-306.

18. Cao X., et al. "Risk subset of the survival for nasopharyngeal carcinoma patients with bone metastases: who will benefit from combined treatment?" Oral Oncology 47.8 (2011): 747752 . 
19. Ong Y., et al. "Design of a prognostic index score for metastatic nasopharyngeal carcinoma". European Journal of Cancer 39.11 (2003): 1535-1541.

20. Zeng L., et al. "Retrospective analysis of 234 nasopharyngeal carcinoma patients with distant metastasis at initial diagnosis: therapeutic approaches and prognostic factors". PLoS One 9.9 (2014): e108070.

21. Chen AY., et al. "Changes in treatment of advanced oropharyngeal cancer, 1985-2001". The Laryngoscope 117.1 (2007): 1621.

22. Haque W., et al. "Radical cystectomy versus chemoradiation for muscle-invasive bladder cancer: impact of treatment facility and sociodemographics". Anticancer Research 37.10 (2017): 5603-5608.

23. Wu L., et al. "Trends in the incidence, treatment and outcomes of patients with intrahepatic cholangiocarcinoma in the USA: facility type is associated with margin status, use of lymphadenectomy and overall survival". World Journal of Surgery 43.7 (2019): 1777-1787.

24. Hauser A., et al. "Impact of academic facility type and volume on post-surgical outcomes following diagnosis of glioblastoma". Journal of Clinical Neuroscience 47 (2018): 103-110.

25. Lagerwaard F., et al. "Identification of prognostic factors in patients with brain metastases: a review of 1292 patients". International Journal of Radiation Oncology* Biology* Physics 43.4 (1999): 795-803.

26. Ruzevick J., et al. "Metastatic squamous cell carcinoma to the brain: an unrecognized pattern of distant spread in patients with HPV-related head and neck cancer". Journal of Neuro-Oncology 112.3 (2013): 449-454.

27. Nayak L., et al. "Epidemiology of brain metastases". Current Oncology Reports 14.1 (2012): 48-54.

28. Chua ML., et al. "Nasopharyngeal carcinoma". The Lancet 387.10022 (2016): 1012-1024.

29. Khanfir A., et al. "Prognostic factors in metastatic nasopharyngeal carcinoma”. Cancer/Radiothérapie 11.8 (2007): 461-464.

30. Kokko L-L., et al. "Significance of site-specific prognosis of cancer stem cell marker CD44 in head and neck squamous-cell carcinoma”. Oral Oncology 47.6 (2011): 510-516.
31. Cooper JS., et al. "National Cancer Database report on cancer of the head and neck: 10-year update". Head and Neck: Journal for the Sciences and Specialties of the Head and Neck 31.6 (2009): 748-758.

32. Zini A., et al. "Oral cancer over four decades: epidemiology, trends, histology, and survival by anatomical sites". Journal of Oral Pathology and Medicine 39.4 (2010): 299-305.

Volume 3 Issue 10 October 2021

(C) All rights are reserved by Wayne D Hsueh., et al. 